\title{
KATARZYNA WASIUTYŃSKA \\ »La misericordia è Gesù Abbandonato«. Riflessione alla luce dell'esperienza spirituale di Chiara Lubich
}

Ho un solo sposo sulla terra: Gesù abbandonato: non ho altro Dio fuori di $\mathrm{Lui}^{1}$ - questa dichiarazione di amore esclusivo scritta da Chiara Lubich (19202008) nel settembre 1949, contiene in sé tutta la ricchezza della sua vita che oggi la Chiesa vuole esaminare per presentarla ai fedeli come via autentica alla santità ${ }^{2}$. Anni dopo, alla domanda sull'essenza della spiritualità del Movimento dei Focolari alla cui base si trova la sua esperienza personale, la Lubich dirà che essa ricorda una medaglia dove su una faccia si trova il grido: Dio mio, Dio mio perché mi hai abbandonato (Mat 27,46), sull'altra la frase della preghiera sacerdotale di Gesù: Che tutti siano uno (Gv 17,21), e in mezzo a essi sta tutto il Vangelo. Nella nostra riflessione che si inserisce nel Giubileo Straordinario vissuto nella Chiesa, guarderemo a Gesù Abbandonato nella prospettiva della misericordia di Dio. Il punto di partenza sono per noi i primi anni della comunità dei Focolari quando Chiara scopriva pian piano il suo Volto particolare e di cui una testimonianza sono le sue lettere degli anni 1943-1949, pubblicate in gran parte da Città Nuova. Una tappa significativa di questa storia spirituale è anche

Katarzyna WASIUTYŃSKA - mgr, doktorantka w Zakładzie Teologii Moralnej, Duchowości i Katolickiej Nauki Społecznej Wydziału Teologicznego Uniwersytetu im. Adama Mickiewicza w Poznaniu, e-mail: katarzyna.wasiutynska@amu.edu.pl

C. Lubich: La dottrina spirituale. Milano 2001 p. 138.

${ }^{2} \mathrm{Nel}$ suo telegramma inviato dopo la morte di Chiara Lubich, Benedetto XVI ringraziava per il suo servizio reso alla Chiesa: un servizio silenzioso e incisivo, in sintonia sempre con il magistero della Chiesa chiamandola generosa testimone di Cristo, donna di intrepida fede, mite messaggera di speranza e di pace (Benedetto XVI: Strumento di Dio. „Città Nuova”. A. 52: 2008 numero speciale p. 28). Il 27 gennaio 2015, il vescovo di Frascati, mons. Raffaello Martinelli, ha aperto in modo solenne il suo processo di beatificazione. 
l'estate del 1949, che fu per Chiara e le prime focolarine un periodo di luce straordinaria a carattere mistico conosciuto come Paradiso $49^{3}$. Poi ci soffermeremo sul modo e sul contenuto della rivelazione di Dio misericordioso in Gesù Abbandonato: su una via che portasse l'uomo all'intimità col Padre e sulla posizione del peccato e della sofferenza in questa prospettiva. Nell'ultima parte prenderemo in considerazione l'atteggiamento esistenziale di Chiara che nasce dall'esperienza vissuta: sulla sua uscita verso il mondo, sulla caratteristica dei rapporti con le persone e con il cosmo.

\section{Alle origini della scoperta di Gesù Abbandonato}

Era il 24 gennaio 1944, a Trento. Un'insegnante ventiquattrenne, SilviaChiara Lubich, da un mese e mezzo consacrata a Dio con un voto perpetuo di castità, chiese a padre Casimiro Bonetti, allora direttore del Terz'ordine francescano, di portare la santa comunione a una sua amica ammalata ${ }^{4}$. Mentre questa pregava in ringraziamento, il religioso chiese inaspettatamente a Chiara in quale momento secondo lei Gesù aveva sofferto di più. Si parlava spesso della sua grande sofferenza nell'orto degli ulivi e fu questo il pensiero spontaneo di questa giovane con una formazione solida nell'Azione Cattolica e nel Terz'ordine francescano. Ma il sacerdote: »Io credo, invece, che sia stato quello in croce, quando ha gridato: 'Dio mio, Dio mio, perché mi hai abbandonato?' (Mat 27,46«. Appena il Padre se ne fu andato, ricorda Doriana Zamboni, avendo udito le parole di Chiara mi rivolsi a lei, sicura d'una spiegazione. Mi disse invece: "Se il più grande dolore di Gesù è stato l'abbandono da parte del Padre suo, noi lo scegliamo come Ideale e lo seguiamo così" ${ }^{5}$.

Questa scelta unica di Gesù Crocifisso e Abbandonato verrà rinnovata più volte da Chiara che confesserà di trovare tutto in Lui e di non conoscere nella sua vita nessun'altro. I primi anni della comunità a Trento che nasceva fra le faccende e le distruzioni della seconda guerra mondiale, erano segnati da una cura particolare dei sofferenti, bisognosi di un sostegno materiale e spirituale, delle vittime delle lotte e delle loro famiglie. In mezzo all'esperienza della fragilità e della vanità del mondo, dietro ogni persona e situazione si nascondeva il volto dell'Abbandonato e l'incontro con Lui era un'occasione per dimostrargli l'amore attraverso dei gesti concreti di misericordia. Lo si avvertiva solo e abbandonato in fondo a tanti cuori umani, nei quali abita per la grazia [...], nelle persone abbandonate a se stesse per la guerra [...], nei dolori e nelle

${ }^{3}$ Cf. C. Lubich: „,Paradiso '49”. „Nuova Umanità”. A. 30: 2008 n. 3 pp. 285-296; V. Araujo: Il patto del '49 nell'esperienza di Chiara Lubich: percorsi interdisciplinari. Roma 2012.

${ }^{4}$ Era Doriana Zamboni. Cf. C. Lubich: La dottrina spirituale, op. cit., pp. 61-62.

${ }^{5}$ Ibidem, p. 61. 
incomprensioni [...], nei poveri che si andavano a cercare, negli ammalati che si visitavano, nei carcerati, in chi aveva errato, come le ragazze madri ${ }^{6}$.

La prima precisa scelta di Gesù Abbandonato marcò l'inizio di un legame d'amore che si era sviluppato, formato e aveva acquistato un significato nuovo nell'arco di tutta la vita. Una settimana sola dopo l'episodio nella casa di Doriana Zamboni descritto sopra, Chiara scriveva alla sua sorella Liliana: Ma soprattutto istruisciti in un libro solo; oh! Se tu mi comprendessi come vorrei io: nel Crocifisso Gesù che fu da tutti abbandonato! Che grida: Dio mio, Dio mio, perché mi hai abbandonato? Oh! Se quel Volto Divino contratto dallo spasimo, quegli occhi arrossati ma che ti guardano con bontà, dimenticando i peccati miei e tuoi che l'hanno così ridotto, fossero sempre davanti al tuo sguardo! [...] Verranno per te le gioie, verranno i dolori e le angosce. Ma se tu in lui ti sforzerai di vedere Gesù, così come te lo presento e sempre te lo presenterò nel culmine del dolore che è il vertice dell'Amore?.

Per Chiara - e dal punto di vista della teologia è un'intuizione giusta - la sofferenza sulla croce si spiega solamente con l'amore che le dona un valore unico, salvifico. Nello stesso tempo rimane in rapporto stretto con i peccati concreti degli uomini che ne sono il motivo, ma proprio in essa vengono dimenticati. Ormai nelle prime lettere di Chiara appare questa consapevolezza della propria colpa alla quale Dio risponde con un eccesso d'amore. Nell'agosto del 1945 scriveva: Lo so: cadrai. Anch'io cado e spesso e sempre. Ma quando alzo lo sguardo a Lui che vedo incapace di vendicarsi perché è fisso in Croce per eccesso d'Amore, mi lascio accarezzare dalla Sua Infinita Misericordia e so che quella sola ha da trionfare in me. A che sarebbe Lui infinitamente Misericordioso? A che? Se non fosse per i nostri peccati? ${ }^{8}$

In modo misterioso il peccato umano attirò Dio il cui amore quale fuoco potente cerca le miserie per divorarle, consumarle ${ }^{9}$. Nell'opera della croce, che per Chiara porta il nome dello Sposo: Gesù Abbandonato, il peccato per quanto suscita una contrizione sincera proveniente dalla fede nell'amore divenne veramente felix culpa, aprendo paradossalmente la via verso Dio. Questo pensiero lo si trova nel modo più esplicito in una lettera a due suore francescane di Rovereto, scritta probabilmente il 3 ottobre 1946: Per unirci a Gesù (unico

\footnotetext{
${ }^{6}$ C. Lubich: L'unità e Gesù Abbandonato. Roma 1984 pp. 70-72.

${ }^{7}$ C. Lubich: Lettere dei primi tempi. Alle origini di una nuova spiritualità. Roma 2010 pp. 31-32.

${ }^{8}$ Ibidem, p. 97.

${ }^{9}$ In una lettera leggiamo: Perché l'anima che ama conosce i gusti dell'Amato e sa che Gesù se è venuto sulla terra, se s'è fatto uomo, se qualcosa brama nel profondo del Suo Cuore Umano Divino è soltanto: Far da Salvatore, Far da Medico! Null'altro desidera. "Fuoco sono venuto a portar sulla terra e che cosa voglio se non che s'accenda? «. È un Fuoco divoratore che ha portato e null'altro vuole che divorare miserie, trovare miserie da consumare! (Ibidem, p. 111).
} 
scopo della nostra vita, specie della nostra ché tutte ci siamo date a Lui) c'è un solo mezzo: $i$ nostri peccati. Occorre levarsi dall'anima ogni altro pensiero. E credere che Gesù è attirato a noi soltanto dall'esposizione umile e confidente e amorosa dei nostri peccati. Noi, per noi, null'altro abbiamo e facciamo che miserie. Lui, per Lui, a riguardo nostro, non ha che una sola qualità: la Misericordia. L'anima nostra si può unire a Lui soltanto offrendoGli in dono, come unico dono, non le proprie virtù, ma $i$ propri peccati! ${ }^{10}$ La miseria dell'uomo diventa una via di comunione con Dio per il fatto che attraverso l'incarnazione e la Pasqua Cristo si identificò con essa, secondo le parole di Paolo: Colui che non aveva conosciuto peccato, Dio lo trattò da peccato in nostro favore, perché noi potessimo diventare per mezzo di lui giustizia di Dio $(2$ Cor 5,21$)$. In realtà troviamo non più il peccato ma il volto del Figlio che donò la sua vita per gli uomini, per amore. La giustificazione si compie in Lui, e non per l'adempimento della Legge per via della virtù.

Quell'incontro con l'amore di Dio che sta alla base di ogni esperienza cristiana $^{11}$ viene vissuto da Chiara soprattutto nella contemplazione di Gesù che chiama Dio mio, Dio mio. In quel grido drammatico ella ritrova la misura massima dell'amore: il dono della vita non solo quella fisica ma anche spirituale che per il Figlio era il legame con il Padre, intimo e irripetibile. Accogliendo nella parte umana le conseguenze del peccato e della morte cioè la voragine che separa la creazione da Dio e il sentimento di una solitudine infinita che ne nasce, le vince in sé con la forza della divinità ${ }^{12}$. Il segno della vittoria in questa lotta interiore sono le parole: Padre, nelle tue mani consegno il mio spirito (Lc 23,46).

\section{Pupilla dell'occhio di Dio: prospettiva trinitaria}

L'anno 1949 segna una tappa nuova nel rapporto con Gesù Abbandonato. Chiara aveva sperimentato che lasciando vivere Lui sul nulla totale della propria esistenza $^{13}$, viene portata insieme a Lui nella vita della Santissima Trinità:

${ }^{10}$ Ibidem

${ }^{11}$ Cf. Benedetto XVI: Lettera enciclica Deus caritas est. Città del Vaticano 2006 n. 1.

12 Affrontando il tema della morte di Gesù nel contesto dell'unità trinitaria, occorre prendere in considerazione la verità sull'unione ipostatica. La Lubich, cosciente di muoversi nell'ambito del mistero, ripete più volte che l'abbandono nella divinità è irreale, rimane però il dramma che tocca la Persona nella sua natura umana. L'opera della riconciliazione e della salvezza avviene necessariamente nell'Uomo-Dio. Nell'abbandono Gesù è, in atto, il Redentore; ̀̀ il Mediatore: fattosi nulla, unisce i figli al Padre (C. Lubich: Il Grido. Roma 2000 p. 25).

${ }^{13}$ In quel periodo Chiara e la comunità dei primi focolarini nascente attorno a lei era animata da un amore particolare per Gesù Abbandonato nel quale scopriva come una sintesi di tutto il Vangelo. Leggiamo nei ricordi scritti nel 1961: Vivendo Gesù Abbandonato si era arrivati a comprendere come Egli s'era annullato e nel nulla stava la nostra vita. Essere con Lui per amore di Lui quel nulla che realmente siamo. Noi nulla, Lui tutto (C. Lubich: „Paradiso'49”, op. cit., p. 286). 
Un giorno [...] Igino Giordani ${ }^{14}$ ed io abbiamo chiesto a Gesù di unire, come Lui sapeva, le nostre anime. E si è sperimentato - per una grazia speciale - cosa significava essere una cellula viva del Corpo mistico di Cristo: era essere Gesù, e come tali in seno al Padre ${ }^{15}$. In Dio può stare solo Dio, scriveva, per quanto siamo dunque innestati pienamente in Gesù Abbandonato, nel quale la Santissima Trinità si spalanca sull'umanità, possiamo secondo il suo disegno eterno rimanere in Dio. Per la natura umana ciò sarebbe stato impossibile, ma la kenosi del Figlio compie questa trasformazione mirabile, innalzando l'uomo al vertice della vita trinitaria: Perché avessimo la Luce, ti venne meno la vista. Perché avessimo l'unione, provasti la separazione dal Padre. Perché possedessimo la sapienza, ti facesti »ignoranza«. Perché ci rivestissimo dell'innocenza, ti facesti "peccato«. Perché Dio fosse in noi, lo provasti lontano da te $e^{16}$.

Questa dinamica dell'amore che dona la propria vita per l'altro, che rinuncia a se stesso perché l'altro possa esistere in pieno, è un riflesso della pericoresi eterna fra Padre, Figlio e Spirito Santo. Solo che nel contesto dello stato di peccato nel quale l'umanità era immersa, sulla croce questo amore era segnato da una grande sofferenza. Tuttavia proprio per il suo compimento nei condizionamenti umani, divenne il destino dell'uomo redento in Cristo. Egli redime, scriveva Chiara nel 1949, quando versa sull'umanità il Divino, attraverso la Ferita dell'Abbandono, che è la pupilla dell'Occhio di Dio sul mondo: un Vuoto Infinito attraverso il quale Dio guarda noi: la finestra di Dio spalancata sul mondo e la finestra dell'umanità attraverso la quale (si) vede $\mathrm{Dio}^{17}$. La pupilla è un foro anatomico, una macchia nera al centro dell'iride che regola l'entrata della luce all'interno dell'occhio. Un tale foro cioè un vuoto, un nulla nella Santissima Trinità divenne il Figlio nel sentimento apparente di una rottura dell'unità con il Padre e in tal modo in Lui la luce della vita divina si dilatò sull'umanità.

La penetrazione da parte dell'uomo della vita intima della Santissima Trinità attraverso grazie abbondanti concesse in Cristo, lo apre a una comprensione dell'amore intriso di misericordia. Si tratta di una prospettiva di sguardo su tutta la creazione: marchiata, per quanto l'uomo lo possa cogliere, dal Creatore stesso. Questa logica particolare viene rivelata in pienezza, nella visione di Chiara,

\footnotetext{
${ }^{14}$ Igino Giordani (1894-1980) - politico, scrittore, giornalista, marito e padre di famiglia. Per il suo contributo allo sviluppo del Movimento dei Focolari ne fu chiamato da Chiara Lubich cofondatore. La sua richiesta rivolta a Chiara di legarsi a lei con un voto di obbedienza per una reciproca santificazione, divenne l'ispirazione per il patto d'unità che segnò il 16.07.1949 l'inizio delle visioni intellettuali nel periodo illuminativo detto Paradiso'49. È in corso il suo processo di beatificazione.

${ }^{15}$ C. Lubich: Il Grido, op. cit., p. 55.

${ }^{16}$ C. Lubich: La dottrina spirituale, op. cit., pp. 137-138.

${ }^{17}$ C. Lubich: Il Grido, op. cit., p. 127.
} 
proprio in Gesù Abbandonato, perché in Lui la sofferenza e l'amore diventano una cosa sola. San Paolo scrive che nel momento culmine del suo abbassamento, che va dall'incarnazione fino alla morte in croce, Egli si fece per noi peccato $^{18}$, maledizione ${ }^{19}$. La sofferenza che prese su di sé contiene in qualche modo tutto il male e l'infelicità del mondo, le conseguenze della disobbedienza e dell'allontanamento dal Creatore.

L'offerta della vita comprende non solo la sua dimensione fisica, ma anche quella spirituale come dimostra il grido sulla croce: Dio mio, Dio mio, perché mi hai abbandonato? (Mat 27,46). La risposta del Padre a quell'amore smisurato è la risuscitazione di Gesù dai morti. Come la redenzione è un'opera trinitaria in cui il mistero di Dio nella sua economia e nella sua comunione interiore di Persone si apre davanti all'uomo, così il dono perfetto del Figlio può essere considerato e spiegato solamente in rapporto al Padre e allo Spirito Santo. L'unica mediazione di Cristo significa che l'uomo redento ha l'accesso a Dio soltanto in Lui e Dio invece guarda, conosce e ama l'umanità nel Figlio. Per la Lubich Egli ha sempre il volto dell'Abbandonato.

\section{Rapporto sponsale: uscita verso il mondo}

Dopo due mesi di un'intensa esperienza mistica avvenuta durante le vacanze nelle Dolomiti, Chiara aveva ormai dimenticato il mondo. Fu allora che Igino Giordani le ricordò chi era lo Sposo della sua vita e le chiese di tornare in città, alla comunità nascente, proprio per la sua scelta esclusiva di Gesù Abbandonato. In quei giorni, tra le lacrime, come lei stessa ricorda, scrisse una sua toccante dichiarazione d'amore che porta la data del 20 settembre 1949:

Ho un solo sposo sulla terra: Gesù abbandonato: non ho altro Dio fuori di Lui. In Lui è tutto il Paradiso con la Trinità e tutta la terra con l'umanità. Perciò il suo è mio e null'altro. E suo è il Dolore universale e quindi mio. Andrò per il mondo cercandolo in ogni attimo della mia vita. Ciò che mi fa male è mio. Mio il dolore che mi sfiora nel presente. Mio il dolore delle anime accanto (è quello il mio Gesù). Mio tutto ciò che non è pace, gaudio, bello, amabile, sereno... ${ }^{20}$

Per Chiara Gesù Abbandonato è lo sguardo amoroso di Dio sull'uomo. Il rapporto sponsale con Lui la spinge a portare lo stesso sguardo su ogni uomo. Pure lei cerca ciò che è misero, sporco, lontano da Dio, perché immedesimata

${ }^{18}$ Colui che non aveva conosciuto peccato, Dio lo trattò da peccato in nostro favore (2 Cor 5,21).

${ }_{19}$ Cristo ci ha riscattati dalla maledizione della legge, diventando lui stesso maledizione per noi, come sta scritto: Maledetto chi pende dal legno $(\mathrm{Ga} 3,13)$

${ }^{20}$ C. Lubich: La dottrina spirituale, op. cit., p. 138. 
con Cristo può partecipare insieme a Lui al compimento dell'opera della salvezza. L'amore puro vuole ad ogni costo bruciare in sé ogni male, per cui è pronta a prendere sulle proprie spalle sia il peccato suo che quello altrui: Ad ogni sbaglio fatto dal fratello chiedo io perdono al Padre come fosse mio ed è mio perché il mio amore se ne impossessa. Così sono Gesù. E sono Gesù Abbandonato sempre di fronte al Padre come Peccato e nel più grande atto d'amore verso i fratelli e quindi verso il Padre. Dunque ogni peccato è mio. Così sono Gesù, Agnello di Dio che toglie i peccati del mondo. Difatti il mio amore li paga, bruciandoli ${ }^{21}$.

La misericordia è una questione di sguardo. E non è uno sguardo che analizza ed esamina per esprimere un giudizio, ma uno sguardo che possiede la forza di cambiare l'altro per estrarre dal suo interno il disegno innato di santità che vi è nascosto dalla creazione. L'uomo che ha sperimentato veramente questo sguardo divino pieno di amore, non può non portarlo agli altri. Quando dopo il vissuto dell'estate 1949, Chiara era tornata a Roma, non voleva guardare quella città distrutta dalla guerra e dalle rovine morali. Non fuggiva però con lo sguardo dalla realtà, ma cercava quella prospettiva di pupilla, di vuoto, per lasciar passare la luce dall'anima unita alla Santissima Trinità. Scriveva: Passo per Roma e non la voglio guardare. [...] Il mio umano si fonde col divino e i miei occhi non sono più spenti, ma, attraverso la pupilla che è vuoto sull'anima, per il quale passa tutta la Luce che è di dentro (se lascio viver Dio in me), guardo al mondo e alle cose [...] Cosicché riaprendo gli occhi sul di fuori vedo l'umanità con l'occhio di Dio che tutto crede perché è Amore ${ }^{22}$.

Questo sguardo contiene in sé il potere di risuscitare la vita nell'altro e d'illuminare il suo volto con la stessa luce soprannaturale. È dunque un amore che non solo non passa indifferente, ma desidera vivificare e incendiare sempre di nuovo, motivare all'azione, scoprire ciò che c'è di prezioso, ma spesso rimane ben nascosto nell'intimo. Sveglia nell'altro la presenza di Dio a condizione però che lei stessa la possieda stabile e forte. Poi da quell'incontro nasce spontanea la necessità di un servizio concreto intrapreso possibilmente nel modo più sapiente, perché sotto l'ispirazione di Cristo: Egli rivede ciechi da illuminare e muti da far parlare e storpi da far camminare. Ciechi alla visione di Dio dentro e fuori di loro. Muti alla Parola di Dio che pure parla in loro e potrebbe da essi esser trasmessa ai fratelli e risvegliarli alla Verità. Storpi immobilizzati, ignari della divina volontà che dal fondo del cuore li sprona al moto eterno che è l'eterno Amore dove trasmettendo Fuoco si viene incendiati ${ }^{23}$. Le opere

\footnotetext{
${ }^{21}$ Testo inedito dell'agosto 1949. Citato in: F. Gillet: La misericordia in Chiara Lubich, cammino di risurrezione. „Unità e carismi”. A. 25: 2015 n. 2 p. 30.

${ }^{22}$ C. Lubich: La dottrina spirituale, op. cit., pp. 218-219. Cf. anche il testo Se il tuo occhio è semplice in: Ibidem, pp. 123-124.

${ }^{23}$ Ibidem, p. 219.
} 
d'amore diventano così una continuazione dell'opera del Figlio fra gli uomini, segni credibili della sua presenza, diffusione del suo regno.

Un tale sguardo ha permesso a Chiara di percorrere in seguito, per più di 50 anni, il mondo con i suoi angoli più bui, più sofferenti (non solo fisicamente, ma anche moralmente e spiritualmente, perché era proprio così il dolore maggiore di Gesù Abbandonato), giacché la misericordia è un amore che prende l'iniziativa. Lei stessa confessava che vivere in modo radicale la spiritualità evangelica di unità, sia nella prima comunità sia più tardi, non sarebbe stato possibile senza una pratica nata all'inizio del Movimento dei Focolari. Si tratta di un proposito solenne di guardarsi gli uni gli altri ogni giorno con occhi nuovi, compiendo durante la notte un'amnistia completa per non ricordare più sbagli o debolezze del passato. Poiché è una cosa naturale che l'amore reciproco perda il suo calore di fronte alle differenze inevitabili nel comportamento e alle piccole incomprensioni, se non lo si coltiva sul modello del Padre che vuole sempre perdonare le colpe, vivendo in un eterno presente. Senza questo atto di perdono quotidiano attuato nel patto di misericordia, come venne chiamato, nessuna comunità sarebbe sopravvissuta e il Movimento stesso, come ebbe a dire una volta Chiara, non si sarebbe diffuso da Trento neppure a Rovereto che dista mezz'ora di viaggio ${ }^{24}$.

La misericordia è, come abbiamo visto, una qualità d'amore che riflette l'eterno dono di sé del Padre e del Figlio nello Spirito Santo. Essa non sta nel coprire o ritoccare l'immagine della realtà. $\mathrm{Al}$ contrario, in Dio essa è la verità, perché nasce dalla purezza dello sguardo. Spiegava la Lubich: Forse quel fratello, come tutti noi, ha commesso degli errori, ma Dio come lo vede? Qual è in realtà la sua condizione, la verità del suo stato? Se è a posto davanti a Dio, Dio non ricorda più nulla, ha tutto cancellato col suo sangue. E noi perché ricordare? Chi è nell'errore in quel momento? Io che giudico, o il fratello? Io. E allora devo mettermi a veder le cose dall'occhio di Dio, nella verità, e trattare in modo conforme col fratello, ché, se per disavventura egli non si fosse ancor sistemato col Signore, il calore del mio amore, che è Cristo in me, lo porterebbe a compunzione come il sole riassorbe e cicatrizza tante piaghe. La carità si mantiene con la verità e la verità è misericordia pura, della quale dobbiamo essere rivestiti da capo a piedi per poterci dire cristiani ${ }^{25}$.

Guardare con lo sguardo di Dio stesso preserva dalla falsità nei rapporti con gli altri. L'amore e la misericordia sono un riflesso della verità sull'essere umano, la garanzia di una conoscenza autentica. La benedizione di un tale comportamento raggiunge non solo la persona a cui si concede di nuovo

${ }^{24}$ Cf. C. Lubich: L'amore reciproco. Roma 2013 p. 89-91; A. Sgariglia: La misericordia nell'eredità spirituale di Chiara Lubich. www.centrochiaralubich.org (28.06.2016).

${ }^{25}$ C. Lubich: La dottrina spirituale, op. cit., p. 144. 
la fiducia e il perdono, ma anche colui che guarda. L'amore ai fratelli è infatti la misura del suo rapporto con Cristo che egli riconosce e accoglie in ogni persona umana. Il cristiano desidera e riesce a vivere in tal modo, poiché prima lui stesso aveva sperimentato questo sguardo pieno di misericordia da parte di Dio. È dunque una conseguenza naturale di essere chiamati all'immagine e somiglianza della Comunione di Persone e condizione di parteciparvi per l'innesto nel Figlio. La Lubich vede in questo amore una via privilegiata alla perfezione cristiana, nella sua dimensione ascetica e mistica ${ }^{26}$.

\section{Implicazioni sociali e nuovo legame con l'universo}

Il cammino per le vie del mondo alla ricerca di Gesù Abbandonato porta alla trasformazione non solo di rapporti singoli con i fratelli. Questa consapevolezza accompagnava Chiara già nei primi anni, quando scriveva: allora tutto si rivoluziona: politica e arte, scuola e religione, vita privata e divertimento [...]. Dio è in noi vivo - se lo facciamo vivere - come legislatore d'ogni legge umana e divina, ché tutta è fattura sua. Ed egli dall'intimo detta ogni cosa, ci insegnaMaestro eterno - l'eterno e il contingente e a tutto dà valore ${ }^{27}$.

Per parlare delle opere di misericordia, la Lubich si riferiva volentieri all'immagine di uno studente che prepara un esame. Così sarà anche del giudizio finale e Dio nel suo sconfinato amore ha già rivelato agli uomini le domande perché ognuna possa prepararvisi in modo migliore. Qualunque sia la nostra vocazione: di padri o di madri, di contadini o di impiegati, di deputati o di capi di Stato, di studenti o di operai, durante il giorno c'è di continuo l'occasione diretta o indiretta di dar da mangiare agli affamati, di istruire gli ignoranti, di sopportare le persone moleste, di consigliare $i$ dubbiosi, di pregare per $i$ vivi e per $i$ morti $^{28}$. La portata e le modalità di azione cambiano a seconda dello stato di vita, del lavoro da svolgere e delle possibilità individuali di ognuno, ma una sola è la ricerca del volto sofferente di Cristo in ogni prossimo. Nello stesso tempo è una via sicura, perché indicata dal Vangelo, per raggiungere la pienezza della vita in Dio, nella dimensione terrena ed escatologica.

La chiamata universale alla santità che si realizza attraverso l'amore concreto, è il primo distintivo, secondo il pensiero e l'esperienza della Lubich, del cristianesimo. Se il disegno di Dio nei confronti del mondo, e soprattutto dei discepoli di Cristo, è perché tutti siano una sola cosa. Come tu, Padre, sei in me $e$ io in te $(\mathrm{Gv} 17,21)$, il segno distintivo di questo legame è la comunione di persone, secondo le parole tutte le cose mie sono tue e tutte le cose tue sono mie $(\mathrm{Gv} 17,10)$. Un dono radicale di sé, di tutto il proprio essere e dei beni

\footnotetext{
${ }^{26}$ Cf. C. Lubich: L'amore al fratello. Roma 2012 p. 71-74.

${ }^{27}$ C. Lubich: La dottrina spiritual, op. cit., p. 220.

${ }^{28}$ Ibidem, p. 122.
} 
in possesso, porta dei frutti sia nell'ambito personale che in quello sociale. La misericordia è una qualità dell'amore universale, che si oltrepassa e cerca l'altra persona per lei stessa. Contiene in sé aspirazioni rivoluzionarie di cambiare l'ambiente e per ciò di natura non può rimanere nascosto, inosservato. Per Chiara il modello irraggiungibile di chi dona la propria vita fisica e spirituale, in vista di tutta l'umanità, è Gesù Abbandonato.

L'opera della redenzione compiuta sulla croce raggiunge in qualche modo anche tutta la creazione, poiché insieme all'uomo colpito dalla sofferenza e dall'abbandono, anche la natura è stata sottomessa alla caducità e geme $e$ soffre fino ad oggi nelle doglie del parto (Ro 8,20.22). All'inizio dell'esperienza di luce straordinaria nel 1949, la Lubich aveva colto in modo particolarmente esplicito nell'universo la presenza di Dio che sostiene tutto nell'esistenza e gli dona la legge perenne di amore reciproco che abbraccia le piante, i fiumi, le stelle ${ }^{29}$. Il Creatore nel suo disegno fece tutto in quanto dono, dove ogni essere si rivolge totalmente verso gli altri. Sulla terra tutto è in rapporto di amore con tutto: ogni cosa con ogni cosa. Occorre però essere l'Amore per trovar il filo d'oro fra gli esseri ${ }^{30}$.

Il contrario di questa esperienza di una presenza potente di Dio fra le creature è il grido di abbandono che risuona tutt'ora. Rispondergli per soddisfarlo può solo chi essendo se stesso l'Amore penetra con la propria esistenza il mondo intero e attribuisce a ogni cosa il valore del dono.

Il rapporto sponsale con Cristo fa sì che l'essere umano si identifichi con ogni sofferenza: non solo spirituale e morale, ma anche quella fisica nelle sue dimensioni cosmiche. Questo desiderio di partecipare all'opera della salvezza è testimoniato dall'invocazione di Chiara:

Signore, dammi tutti i soli...

Ho sentito nel mio cuore la passione che invade il tuo per tutto l'abbandono in cui nuota il mondo intero.

Amo ogni essere ammalato e solo:

anche le piante sofferenti mi fanno pena..., anche gli animali soli [...].

${ }^{29}$ Scriveva la Lubich degli inizi dell'esperienza del Paradiso'49: Ricordo che in quei giorni la natura mi sembrava tutta avvolta dal sole; già lo era dal lato fisico, ma a me sembrava che un sole più forte la avvolgesse, la inzuppasse, cosicché mi appariva tutta «innamorata». Vedevo le cose, i fiumi, le piante, i prati, le erbe, fra loro legati da un legame d'amore nel quale ognuno aveva un perché d'amore versi gli altri (C. Lubich: „Paradiso'49”. op. cit. p. 287). Cf. anche F. Ciardi: La parola come amore e la presenza di Dio sotto le cose. Lettura trinitaria di un'esperienza. „Nuova Umanità”. A. 28: 2006 n. 2 pp. 155-180.

${ }^{30}$ C. Lubich: La dottrina spirituale, op. cit., p. 130. 
Dammi, mio Dio, d'essere nel mondo il sacramento tangibile del tuo Amore, del tuo essere Amore: d'esser le braccia tue che stringono a sé e consumano in amore tutta la solitudine del mondo ${ }^{31}$.

La misericordia che riempie il Cuore di Gesù è nella comprensione della Lubich simile a un fuoco che divora la disunità, la sofferenza e ogni male. Solo un tale amore può rispondere a una nostalgia inconsolabile di tutta la creazione e Chiara si sente chiamata a esserne un segno concreto grazie all'immedesimazione con Gesù Abbandonato nel momento culmine della sua rivelazione in quanto Uomo-Dio. Chi vive con Lui e per Lui non troverà la pace finché tutto l'universo non verrà accolto fra le braccia misericordiose del Salvatore, poiché tutto fu da Dio creato per essere un riflesso dell'amore trinitario.

I pensieri presentati sopra costituiscono solo un primo tentativo di aprire piste da percorrere per una riflessione più approfondita sulla misericordia negli scritti di Chiara Lubich segnati dal timbro dell'unità. Dove sta la sua originalità e la novità che ogni carisma introduce nella storia spirituale della Chiesa? Prima di tutto ci pone davanti Gesù Abbandonato in quanto volto misericordioso di Dio che apre il cuore alla miseria umana e cerca Lui stesso di rimediarvi in modo pieno e perfetto. Gesù Abbandonato è il mistero dell'unità, è la via della comunione degli uomini con Dio e fra di loro. L'accoglienza della sua logica d'amore fino al dono della vita rende capaci d'inserirsi nell'attualizzazione del disegno di Dio nei confronti dell'uomo e dell'universo.

Le profonde intuizioni della Lubich, il cui spessore va misurato e valorizzato attraverso la sua propria vita spesa in una donazione fedele e radicale a Gesù Abbandonato, risuonano oggi con una grande forza. La spiritualità dell'uomo odierno non può permettersi il lusso di tacere tutti i "perché" gridati in modo drammatico verso quel Dio che più che mai si vuole credere e conoscere come amore. Quella domanda sulla croce svela un'altra dimensione dell'essere divino in quanto dono smisurato di sé che ridefinisce la forma e la giustizia di tutta la realtà. Per poter guardare quest'ultima secondo la verità, occorre uno sguardo diverso, rinnovato da un amore che ha in sé il coraggio di farsi uno spazio vuoto e buio. Per Chiara la pupilla è Gesù Abbandonato.

\section{Bibliografia}

Araujo V.: Il patto del «49 nell»esperienza di Chiara Lubich : percorsi interdisciplinari. Roma 2012.

\footnotetext{
${ }^{31}$ Ibidem, pp. 124-125.
} 
Benedetto XVI: Strumento di Dio. „Città Nuova”. A. 52: 2008 numero speciale p. 28.

Benedetto XVI: Lettera enciclica Deus caritas est. Città del Vaticano 2006.

Ciardi F.: La parola come amore e la presenza di Dio sotto le cose. Lettura trinitaria di un'esperienza. „Nuova Umanità”. A. 28: 2006 n. 2 pp. 155-180.

Gillet F.: La misericordia in Chiara Lubich, cammino di risurrezione. „Unità e carismi”. A. $25: 2015$ n. 2 p. $28-33$.

Lubich C.: Il Grido. Roma 2000.

Lubich C.: L'amore al fratello. Roma 2012.

Lubich C.: L'amore reciproco. Roma 2013.

Lubich C.: L'unità e Gesù Abbandonato. Roma 1984.

Lubich C.: La dottrina spirituale. Milano 2001.

Lubich C.: Lettere dei primi tempi. Alle origini di una nuova spiritualità. Roma 2010.

Lubich C.: „Paradiso '49”. „Nuova Umanità”. A. 30: 2008 n. 3 pp. 285-296.

Sgariglia A.: La misericordia nell'eredità spirituale di Chiara Lubich. www.centrochiaralubich.org (28.06.2016)

\section{STRESZCZENIE \\ »Milosierdzie to Jezus Opuszczony«. Refleksja w świetle doświadczenia duchowego Chiary Lubich}

W tajemniczym wołaniu Jezusa na krzyżu: „Boże mój, Boże mój, czemuś Mnie opuścił?” (Mk 15, 34) Chiara Lubich (1920-2008) odczytuje najpełniejsze objawienie miłości i miłosierdzia Boga. Jako młoda dziewczyna wybiera Opuszczonego jako ideał swojego życia, jedynego Oblubieńca, a cennym świadectwem tego pierwszego okresu są listy z lat 1943-1949. Lato 1949 przynosi jej głębokie, mistyczne doświadczenie, w którym przenika ona tajemnice Boga Trójjedynego dzięki szczególnej jedności z Synem. Jej powrót do świata naznaczony będzie odtąd pragnieniem, by to samo doświadczenie przenieść na relacje $\mathrm{z}$ innymi ludźmi, $\mathrm{z}$ całym stworzeniem. Dla Chiary uczestniczyć w miłosierdziu Boga to posiadać Jego czyste spojrzenie, które każde napotkane cierpienie i grzech przemienia w miłość.

Słowa kluczowe: Chiara Lubich, miłosierdzie, Jezus Opuszczony, duchowość.

\section{SUMMARY \\ »Mercy is Jesus Forsaken«. Reflection in the Light of the Spiritual Experience of Chiara Lubich}

In the mysterious Jesus' cry on the cross: "My God, my God, why have you forsaken me?" (Mark 15, 34) Chiara Lubich (1920-2008) reads the fullest revelation of God's love and mercy. As a young woman, she chooses Jesus Forsaken as the ideal of her life, the only Spouse; a precious testimony of this first period are her letters of the years 1943-1949. The summer of 1949 brings her a deep, mystical experience in which she penetrates the mystery of triune God thanks to a particular unity with the Son. Her return to the world will henceforth be marked with a desire to transfer the same experience to the relationships with other people and the whole creation. For Chiara to participate in the mercy of God is to have his pure look which transforms every encountered suffering and sin into love.

Keywords: Chiara Lubich, mercy, Jesus Forsaken, spirituality. 